\title{
Clinical Relevance of Targeting the Gastrin-Releasing Peptide Receptor, Somatostatin Receptor 2, or Chemokine C-X-C Motif Receptor 4 in Breast Cancer for Imaging and Therapy
}

\author{
Simone U. Dalm¹, Anieta M. Sieuwerts², Maxime P. Look ${ }^{2}$, Marleen Melis ${ }^{1}$, Carolien H.M. van Deurzen ${ }^{3}$, \\ John A. Foekens ${ }^{2}$, Marion de Jong ${ }^{1}$, and John W.M. Martens ${ }^{2}$ \\ ${ }^{1}$ Departments of Nuclear Medicine and Radiology, Erasmus MC, Rotterdam, The Netherlands; ${ }^{2}$ Department of Medical Oncology and \\ Cancer Genomics Netherlands, Erasmus MC Cancer Institute, Erasmus MC, Rotterdam, The Netherlands; and ${ }^{3}$ Department of \\ Pathology, Erasmus MC, Rotterdam, The Netherlands
}

Imaging and therapy using radioligands targeting receptors overexpressed on tumor cells is successfully applied in neuroendocrine tumor patients. Because expression of the gastrin-releasing peptide receptor (GRPR), somatostatin receptor 2 (SSTR2), and chemokine C-X-C motif receptor 4 (CXCR4) has been demonstrated in breast cancer, targeting these receptors using radioligands might offer new imaging and therapeutic opportunities for breast cancer patients. The aim of this study was to correlate messenger RNA (mRNA) expression of GRPR, SSTR2, and CXCR4 with clinicopathologic and biologic factors, and with prognosis and prediction to therapy response, in order to identify specific breast cancer patient groups suited for the application of radioligands targeting these receptors. Methods: First, we studied GRPR and SSTR2 expression in 13 clinical breast cancer specimens by in vitro autoradiography and correlated this with corresponding mRNA levels to investigate whether mRNA levels reliably represent cell surface expression. Next, GRPR, SSTR2, and CXCR4 mRNA levels were measured by quantitative reverse transcriptase polymerase chain reaction in 915 primary breast cancer tissues and correlated with known clinicopathologic and biologic factors, disease-free survival, distant metastasis-free survival, and overall survival (DFS, MFS, and OS, respectively). In 224 adjuvant hormonal treatment-naïve estrogen receptor (ER, ESR1)-positive patients who received tamoxifen as first-line therapy for recurrent or metastatic disease, the expression levels of the receptors were correlated with progression-free survival. Results: Our results showed a significant positive correlation between GRPR and SSTR2 expression analyzed by in vitro autoradiography and by quantitative reverse transcriptase polymerase chain reaction (Spearman's rank correlation coefficient $\left[R_{s}\right]=$ $0.94, P<0.001$, and $\mathrm{R}_{\mathrm{s}}=0.73, P=0.0042$, respectively). Furthermore, high GRPR and SSTR2 mRNA levels were observed more frequently in ESR1-positive specimens, whereas high CXCR4 expression was associated with ESR1-negative specimens. Also, high mRNA expression of CXCR4 was associated with a prolonged DFS, MFS, and OS (multivariate hazard ratio MFS $=0.76[95 \%$ confidence interval, 0.64-0.90], $P=0.001$ ), whereas high mRNA levels of GRPR were associated with a prolonged progression-free survival after the start of first-line tamoxifen treatment (multivariate hazard ratio $=0.68$ [95\% confidence interval, 0.48-0.97], $P=$ 0.031). Conclusion: Our data indicate that imaging and therapy

\footnotetext{
Received May 14, 2015; revision accepted Jul. 21, 2015.

For correspondence or reprints contact: Simone Dalm, Rm. Na620, P.O Box 2040, 3000 CA Rotterdam, The Netherlands.

E-mail: s.dalm@erasmusmc.nl

Published online Aug. 6, 2015

COPYRIGHT (c) 2015 by the Society of Nuclear Medicine and Molecular Imaging, Inc.
}

using GRPR or SSTR2 radioligands might especially be beneficial for ESR1-positive breast cancer and CXCR4 radioligands for ESR1negative breast cancer.

Key Words: breast cancer; GRPR; SSTR2; CXCR4; PRS/PRRT

J Nucl Med 2015; 56:1487-1493

DOI: 10.2967/jnumed.115.160739

\section{B} reast cancer is the most common cancer found in women worldwide. An estimated 1.7 million new cases were diagnosed in 2012 worldwide, and 522,000 people died as a consequence of the disease, making it the fifth cause of death by cancer overall (1).

Multiple subtypes of breast cancer exist, with different molecular characteristics such as the absence or presence of estrogen receptor (ER, ESRl), progesterone receptor $(\mathrm{PR}, P G R)$, and human epidermal growth factor 2 (HER2, ERBB2) (2). In the case of ER and HER2, these receptors also serve as therapeutic targets. ER-positive patients are treated with either aromatase inhibitors or ER antagonists, most commonly tamoxifen, whereas HER2-positive patients are often treated with the HER2-specific monoclonal antibody trastuzumab (2). However, in the recurrent or metastatic setting nearly all patients acquire resistance against tamoxifen and trastuzumab after an initial response $(3,4)$.

Mammography is the standard method used for breast cancer screening, in some cases supplemented with MR imaging or ultrasound (5). Unfortunately these methods may lead to falsepositive and false-negative results $(6,7)$. Because current imaging and the above-mentioned therapy options, in particular, have limitations and are not always successful, new imaging and therapeutic options are urgently needed.

Peptide receptor scintigraphy and peptide receptor radionuclide therapy are methods based on targeting receptors overexpressed on tumor cells using radioligands for diagnostic and therapeutic purposes. Within nuclear medicine, radiolabeled somatostatin (SST) analogs are most widely and successfully used for the localization, treatment, and evaluation of neuroendocrine tumors (8). These SST analogs bind to SST receptors (SSTR, especially SSTR2) overexpressed on tumor cells, enabling imaging when labeled with $\gamma$ or positron emitters and therapy when labeled with $\beta$ - or $\alpha$-particle emitters. Currently, multiple radiolabeled SST analogs targeting SSTR2 are available and used in the clinic (9). 
In the past decade, imaging of breast cancer patients using SSTR2 radioligands has been studied with varying results $(10,11)$. Currently, considerably improved SSTR2-directed radiotracers and imaging equipment are available.

Other promising targeting radioligands for breast cancer comprise radiolabeled gastrin-releasing peptide (GRP) analogs, earlier applied for the visualization and therapy of prostate cancer lesions, because significant GRP receptor (GRPR) levels are present in most primary prostate cancer tissues (12-14). Previous studies by Reubi et al. (15) showed a high expression of both SSTR2 and GRPR in breast cancer. SSTR2 and high-density GRPR expression was found in $75 \%$ and $74 \%$ of breast cancer cases, respectively.

Moreover, chemokine C-X-C motif receptor 4 (CXCR4) expression has been reported in most breast cancers. In a study by Salvucci et al. (16), in which 2,022 breast cancer specimens were analyzed for CXCR4 expression using immunohistochemistry, 67\% of invasive tumors showed high nuclear staining and $41 \%$ of tumors showed cytoplasmic staining (12). Promising radiolabeled peptide derivatives binding to CXCR4 have been synthesized to target these receptors $(17,18)$. So ${ }^{68} \mathrm{Ga}$-pentaxifor, a CXCR4 radioligand, has successfully been used in a clinical study for the imaging of multiple myeloma patients (19). Thus, these 3 promising categories of radiolabeled compounds could be of promise in breast cancer patients.

Until now, little was known about the correlation between GRPR, SSTR2, and CXCR4 expression levels in breast cancer lesions and important molecular and prognostic characteristics, such as hormone receptor expression, as well as the association of GRPR, SSTR2, and CXCR4 expression with disease-free survival, distant metastasis-free survival, or overall survival (DFS, MFS, and OS, respectively) and with progression-free survival (PFS) after endocrine treatment.

In this study, we first analyzed the correlation between messenger RNA (mRNA) levels and protein expression of GRPR and SSTR2. Subsequently, we analyzed the mRNA expression of GRPR, SSTR2, and CXCR4 in human breast cancer specimens. The aims of this study were to correlate $G R P R, S S T R 2$, and CXCR4 mRNA expression levels with clinicopathologic and biologic factors as well as with prognosis and outcome on tamoxifen therapy, to assess the potential impact of radioligands targeting these receptors for imaging and therapeutic purposes in breast cancer, and to thereby identify patient subgroups that potentially would benefit from application of these radiopharmaceuticals.

\section{MATERIALS AND METHODS}

\section{Human Breast Cancer Cases}

The study (MEC02.953) was approved by the Erasmus MC Medical Ethical Committee and adhered to the Code of Conduct of the Federation of Medical Scientific Societies in The Netherlands.

The primary breast cancer tissue of 915 female patients (mean age \pm $\mathrm{SD}, 58 \pm 13$ y) (684 M0 [no metastasis at diagnosis] lymph-nodenegative [LNN], 194 M0 lymph-node-positive [LNP], 24 M1 LNP, and 13 patients with unknown nodal status at time of primary treatment) who visited the clinic between 1979 and 2000 were selected from the Erasmus MC fresh-frozen tissue bank as described before (20). The inclusion criteria and the determination of clinicopathologic and biologic factors are described in the supplemental data (supplemental materials are available at http://jnm.snmjournals.org). GRPR, $S S T R 2$, and CXCR4 expression was initially correlated with clinicopathologic and biologic factors in the LNN M0 patient group $(n=$ 194). A representative group of LNP tumors $(n=194)$ was added to study the influence of positive nodal status on the correlation analyses.
For prognosis, we focused our analyses on the cohort of 684 systemic treatment-naive patients with $\mathrm{LNN}$ disease; for prediction of therapy response, a cohort of 224 hormonal treatment-naive ER-positive patients who received tamoxifen as first-line therapy for recurrent or metastatic disease was analyzed. The clinicopathologic and biologic factors of the LNN M0 tumors are shown in Table 1, and clinicopathologic and biologic factors for the LNN and LNP M0 patient group and the ER-positive first-line tamoxifen-treated subcohort are shown in Supplemental Tables 1A and 1B, respectively. Patients were censored at 120-mo follow-up after surgical removal of the primary tumor in the regression analysis for DFS (283 events), MFS (241 events), and OS (223 events) and at $36 \mathrm{mo}$ after the start of tamoxifen treatment for analysis of PFS (24 events). The study design is depicted in Figure 1.

\section{RNA Isolation, Complementary DNA Synthesis, and Quantitative Reverse Transcriptase Polymerase Chain Reaction (RT-qPCR)}

Tissue processing, RNA isolation, complementary DNA synthesis, and RT-qPCR were performed and normalized using the $\delta \mathrm{Cq}$ method on the average of 3 reference genes (HMBS, HPRT1, and TBP) as described (21). All RNA samples that required more than 25 rounds of real-time PCR for detectable products of our 3 reference genes at a fixed input of $10 \mathrm{ng}$ of total RNA and at a threshold of 0.1 were considered of insufficient quality and were excluded from further analysis. Target genes were quantified using the following intron-spanning Taqman probe-based gene expression assays (Applied BioSystems/Life Technologies): GRPR, Hs01055872 m1; SSTR2, Hs0099356 m1; and CXCR4, Hs00237052 $\mathrm{ml}$, according to the manufacturer's instructions in a MX3000P Real-Time PCR System (Agilent). Genomic grade index (GGI), a gene expression pattern of histologic tumor grade, and ESR1, $P G R$, and $E R B B 2$ levels and status of the samples were already known based on quantification as previously described (22-24).

\section{Radioligands and In Vitro Autoradiography}

Peptide analogs targeting the SSTR2 and GRPR, DOTA-Tyr ${ }^{3}$ octreotate (Mallinckrodt) and AMBA (BioSynthema), respectively, were radiolabeled with ${ }^{111}$ In (Covidien), as previously described (25). Quenchers (10 mM methionine, $3.5 \mathrm{mM}$ ascorbic acid, and $3.5 \mathrm{mM}$ gentisic acid) were used to prevent radiolysis (26). Specific activity of both radiotracers was $50 \mathrm{MBq} / \mathrm{nmol}$. Radiometal incorporation $(>99 \%)$ and radiochemical purity $(>90 \%)$ were measured by instant thin-layer chromatography on silica gel and high-pressure liquid chromatography as previously described (26).

The CXCR4 radioligand, pentaxifor, available to us showed reduced receptor affinity when radiolabeled with ${ }^{111} \mathrm{In}$, and thus satisfying in vitro autoradiography studies using this compound could not be performed.

In the in vitro autoradiography assay, tissue sections of 13 fresh-frozen breast cancer specimens $(10 \mu \mathrm{m})$ were incubated with $10^{-9} \mathrm{M}$ ${ }^{111} \mathrm{In}$-AMBA and ${ }^{111} \mathrm{In}$-DOTA-Tyr ${ }^{3}$-octreoate for $1 \mathrm{~h}$, without and with $10^{-6} \mathrm{M}$ unlabeled tracer as control for nonspecific binding. H69 (SSTR2positive, GRPR-negative) and PC3 xenografts (GRPR-positive, SSTR2negative) were used as controls. Results were quantified using OptiQuant software (Perkin Elmer), and the net percentage binding of added dose was calculated. The in vitro autoradiography assay and quantification of the results are described in more detail in the supplemental data.

\section{Statistics}

Statistical analyses are described in the supplemental data.

\section{RESULTS}

\section{In Vitro Autoradiography and Correlation with mRNA Expression}

Specific binding to tumor cells of the GRPR- and SSTR2-mediated radiotracers, ${ }^{111} \mathrm{In}$-AMBA and ${ }^{111} \mathrm{In}$-DOTA-Tyr ${ }^{3}$-octreotate, 
TABLE 1

Associations of GRPR, SSTR2, and CXCR4 mRNA Levels in LNN MO Patients

\begin{tabular}{|c|c|c|c|c|c|c|c|c|}
\hline \multirow[b]{2}{*}{ Characteristic } & \multirow[b]{2}{*}{$\begin{array}{c}\text { No. of } \\
\text { patients }\end{array}$} & \multirow[b]{2}{*}{$\begin{array}{l}\text { Percentage } \\
\text { of patients }\end{array}$} & \multicolumn{2}{|c|}{ GRPR mRNA $\left(\times 10^{-2}\right)$} & \multicolumn{2}{|c|}{ SSTR2 mRNA $\left(\times 10^{-2}\right)$} & \multicolumn{2}{|c|}{ CXCR4 mRNA $\left(\times 10^{-2}\right)$} \\
\hline & & & Median & $\begin{array}{l}\text { Interquartile } \\
\text { range }\end{array}$ & Median & $\begin{array}{l}\text { Interquartile } \\
\text { range }\end{array}$ & Median & $\begin{array}{l}\text { Interquartile } \\
\text { range }\end{array}$ \\
\hline All patients in this cohort & 684 & $100 \%$ & 0.72 & 7.07 & 0.58 & 1.75 & 11.78 & 13.13 \\
\hline \multicolumn{9}{|l|}{ Age at surgery $(\mathrm{y})$} \\
\hline$\leq 40$ & 60 & $9 \%$ & 1.17 & 12.72 & 0.90 & 2.99 & 14.06 & 13.75 \\
\hline $41-55$ & 252 & $37 \%$ & 0.97 & 9.20 & 0.61 & 1.64 & 11.58 & 13.18 \\
\hline $56-70$ & 218 & $32 \%$ & 0.52 & 5.38 & 0.52 & 1.68 & 12.19 & 11.34 \\
\hline$>70$ & 154 & $23 \%$ & 0.72 & 4.44 & 0.62 & 1.61 & 9.99 & 12.98 \\
\hline$P^{\dagger}$ & & & 0.52 & & 0.68 & & 0.0403 & \\
\hline \multicolumn{9}{|l|}{ Menopausal status } \\
\hline Premenopausal & 273 & $40 \%$ & 1.26 & 10.95 & 0.62 & 1.82 & 11.81 & 13.73 \\
\hline Postmenopausal & 411 & $60 \%$ & 0.60 & 4.87 & 0.55 & 1.57 & 11.76 & 12.12 \\
\hline$P^{\dagger}$ & & & 0.13 & & 0.53 & & 0.39 & \\
\hline \multicolumn{9}{|l|}{ Surgery } \\
\hline Lumpectomy & 378 & $55 \%$ & 0.61 & 7.69 & 0.57 & 1.82 & 11.67 & 13.15 \\
\hline Ablation & 306 & $45 \%$ & 0.90 & 6.79 & 0.60 & 1.56 & 11.90 & 13.00 \\
\hline$P^{\dagger}$ & & & 0.69 & & 0.59 & & 0.65 & \\
\hline \multicolumn{9}{|l|}{ Pathologic tumor size } \\
\hline pT1 & 307 & $45 \%$ & 1.25 & 8.54 & 0.69 & 1.87 & 12.03 & 13.40 \\
\hline pT2 + unknown & 351 & $51 \%$ & 0.41 & 5.25 & 0.51 & 1.65 & 11.53 & 12.84 \\
\hline pT3 + pT4 & 26 & $4 \%$ & 0.58 & 3.05 & 0.50 & 1.38 & 12.19 & 13.53 \\
\hline$P^{\dagger}$ & & & 0.0014 & & 0.24 & & 0.92 & \\
\hline \multicolumn{9}{|l|}{ ESR1 mRNA status ${ }^{\ddagger}$} \\
\hline Negative $<0.2$ & 184 & $27 \%$ & 0.09 & 0.13 & 0.28 & 0.42 & 14.74 & 13.83 \\
\hline Positive $\geq 0.2$ & 500 & $73 \%$ & 2.46 & 10.98 & 0.81 & 2.59 & 10.98 & 12.16 \\
\hline$P^{\ddagger}$ & & & $<0.001$ & & $<0.001$ & & $<0.001$ & \\
\hline \multicolumn{9}{|l|}{ PGR mRNA status ${ }^{\ddagger}$} \\
\hline Negative $<0.1$ & 285 & $42 \%$ & 0.12 & 0.32 & 0.32 & 0.56 & 14.36 & 14.31 \\
\hline Positive $\geq 0.1$ & 399 & $58 \%$ & 3.67 & 12.68 & 1.02 & 2.98 & 10.45 & 11.09 \\
\hline$P^{\ddagger}$ & & & $<0.001$ & & $<0.001$ & & $<0.001$ & \\
\hline \multicolumn{9}{|l|}{ ERBB2 $\mathrm{mRNA}$ status ${ }^{\ddagger}$} \\
\hline Negative $<18$ & 574 & $84 \%$ & 0.99 & 8.28 & 0.61 & 1.92 & 11.64 & 12.96 \\
\hline Positive $\geq 18$ & 107 & $16 \%$ & 0.30 & 1.51 & 0.49 & 1.00 & 13.88 & 13.32 \\
\hline$P^{q}$ & & & $<0.001$ & & 0.0344 & & 0.22 & \\
\hline \multicolumn{9}{|l|}{ Grade (GGI) } \\
\hline 1 & 227 & $33 \%$ & 2.42 & 10.46 & 0.75 & 2.11 & 10.83 & 11.03 \\
\hline 2 & 229 & $33 \%$ & 0.89 & 6.92 & 0.63 & 2.41 & 11.44 & 14.59 \\
\hline 3 & 224 & $33 \%$ & 0.13 & 1.42 & 0.34 & 0.98 & 13.83 & 13.58 \\
\hline$P$ था & & & $<0.001$ & & $<0.001$ & & $<0.001$ & \\
\hline \multicolumn{9}{|l|}{$\begin{array}{l}\text { Percentage } \\
\text { invasive tumor cells }\end{array}$} \\
\hline$\leq 70 \%$ & 470 & $69 \%$ & 0.81 & 6.84 & 0.63 & 1.88 & 12.57 & 13.92 \\
\hline$>70 \%$ & 214 & $31 \%$ & 0.64 & 8.28 & 0.43 & 1.28 & 9.13 & 10.70 \\
\hline$P^{\dagger}$ & & & 0.87 & & 0.002 & & $<0.001$ & \\
\hline
\end{tabular}

*Because of missing numbers, not all categories add up to 684 .

${ }^{\dagger} P$ for Mann-Whitney $U$ or Kruskal-Wallis test when appropriate.

${ }^{\ddagger} E S R 1, P G R$, and ERBB2 were determined by real-time PCR; cut points were as follows: $E S R 1=0.2, P G R=0.1$, and $E R B B 2=18.0$ (mRNA level relative to reference gene set).

${ }^{\text {I }} P$ for Spearman rank-correlation test. 


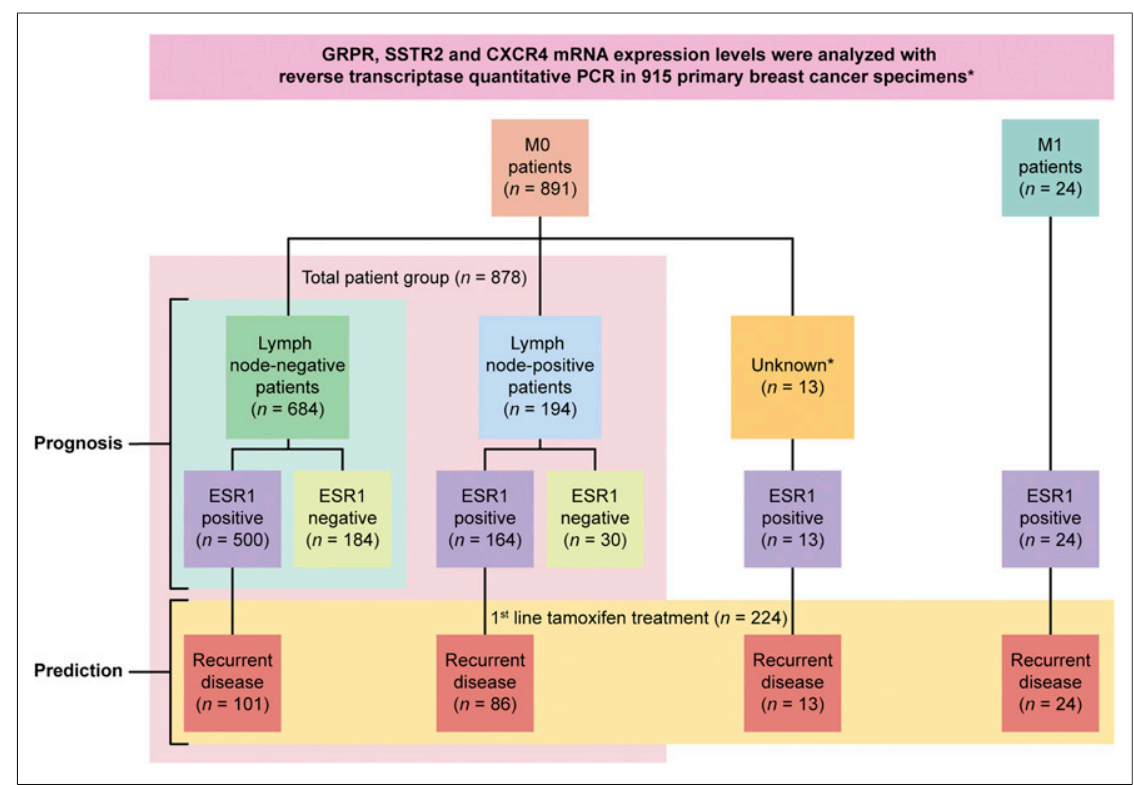

FIGURE 1. Study design. mRNA expression levels of GRPR, SSTR2, and CXCR4 of 915 primary breast cancer specimens (684 MO LNN, 194 MO LNP, 13 with unknown nodal status, and 24 M1) were analyzed using RT-qPCR. LNN and LNP MO patient groups were used to study association of GRPR, SSTR2, and CXCR4 expression and clinicopathologic and biologic factors, with focus on MO LNN patient group. Association of GRPR, SSTR2, and CXCR4 with prognostic factors was studied in MO LNN patients. mRNA levels of ER-positive primary tumors of patients with recurrent breast cancer who received first-line tamoxifen treatment were used to study association of GRPR, SSTR2, and CXCR4 mRNA expression and PFS.

respectively, was demonstrated using in vitro autoradiography on 13 selected human breast cancer specimens with varying levels of mRNA receptor expression. Two mouse xenografts served as positive and negative control (Fig. 2A). Autoradiography results were quantified and correlated with the level of mRNA expression of the respective receptors, resulting in a significant positive correlation for both GRPR (Spearman's rank correlation coefficient $\left.\left[\mathrm{R}_{\mathrm{s}}\right]=\mathrm{R}_{\mathrm{s}}=0.94, P<0.0001\right)$ and SSTR2 $\left(\mathrm{R}_{\mathrm{s}}=0.73, P=\right.$ 0.0042) (Fig. 2B). Furthermore, binding of the tracers was observed only on tumor cells and not on the surrounding stromal cells. We thus concluded that mRNA expression for GRPR and $S S T R 2$ can be used as a predictor for binding of the radiotracers to tumor tissue.

\section{Correlation of GRPR, SSTR2, and CXCR4 mRNA Expression with Clinicopathologic and Biologic Factors}

We focused on the $684 \mathrm{LNN} \mathrm{M0}$ patients to study the correlation between GRPR, SSTR2, and CXCR4 mRNA levels and known clinicopathologic and biologic factors. The results of the correlation analyses are shown in Table 1 . To study the influence of positive nodal status on the correlation analyses, a representative group of 194 LNP M0 tumors were added to the study. Results of the LNN and LNP M0 patient group are described in Supplemental Table 1A.

A significant correlation was observed between $G R P R$ mRNA levels and a smaller pathologic tumor size $(P=0.0014)$, a positive ESR1 $(P<0.001)$ and $P G R$ status $(P<0.001)$, a negative ERBB2 $(P<0.001)$ status, and a favorable GGI $(P<0.001)$.

SSTR 2 mRNA expression showed a significant correlation with a positive $E S R 1(P<0.001)$ and $P G R$ mRNA status $(P<0.001)$, a negative $E R B B 2$ status $(P=0.0344)$, favorable GGI $(P<$ $0.001)$, and $70 \%$ or less invasive tumor cells $(P=0.002)$.
CXCR4 mRNA expression showed a significant negative correlation with ESRl $(P<0.001)$ and $P G R$ mRNA status $(P<$ $0.001)$ and was associated with an unfavorable GGI $(P<0.001)$. Furthermore, $C X C R 4$ mRNA levels were higher in tumors with $70 \%$ or less invasive tumor cells $(P<$ $0.001)$.

\section{Association of GRPR, SSTR2, and CXCR4 mRNA Expression with Prognosis and Efficacy of Tamoxifen Treatment}

To exclude the possible confounding effect of adjuvant therapy on prognosis, the association of GRPR, SSTR2, and $C X C R 4$ expression with prognosis was evaluated in the LNN patient group, which did not receive adjuvant systemic therapy. The results of the evaluation of GRPR, SSTR2, and $C X C R 4$ mRNA expression with DFS, MFS, and OS are shown in Supplemental Table 2.

No significant associations were observed between GRPR and SSTR2 mRNA expression and DFS, MFS, or OS. For CXCR4, however, there was a significant association of its expression with a favorable DFS, MFS, and OS, both when analyzed as a continuous variable and when dichotomized at the median level. For the primary endpoint MFS, the results of the multivariate analysis were hazard ratio $(\mathrm{HR})=0.76(95 \%$ confidence interval $[\mathrm{CI}], 0.64-0.90), P=$ 0.001 , when analyzed as a continuous variable, and $\mathrm{HR}=0.71$ (95\% CI, 0.55-0.91), $P=0.011$, when dichotomized at the median level.

To visualize the association of the levels of $C X C R 4$ mRNA with MFS, Kaplan-Meier analysis was performed as a function of the quartile levels of CXCR4 mRNA (Fig. 3). The results show a clear trend of quartiles, with lower expression having a worse MFS time.

In addition, GRPR, SSTR2, and CXCR4 mRNA expression levels were correlated with the efficacy of tamoxifen treatment in ESRIpositive patients with recurrent disease (Supplemental Table 1B). There was a significant correlation between high GRPR mRNA levels and prolonged PFS after the start of first-line tamoxifen treatment, indicating that GRPR expression has predictive value for the efficacy of tamoxifen therapy (Fig. 4; Supplemental Table 3) (25\% high vs. $75 \%$ low, univariate $\mathrm{HR}=0.65$ [95\% CI, 0.47-0.91], $P=0.011$, and multivariate $\mathrm{HR}=0.68$ [95\% CI, 0.48-0.97], $P=0.031$ ).

\section{DISCUSSION}

We have analyzed GRPR, SSTR2, and CXCR4 mRNA expression in 915 primary breast cancer tissues and correlated mRNA expression of these receptors with clinicopathologic and biologic factors and with prognosis and prediction to therapy response, to study the relevance of the application of radioligands targeting these receptors for imaging and therapy in breast cancer patients. For this, we first successfully demonstrated in vitro binding of radiotracers for GRPR and SSTR2 to tissue sections and showed a significant positive correlation between radiotracer binding and mRNA expression, demonstrating that mRNA levels of these 


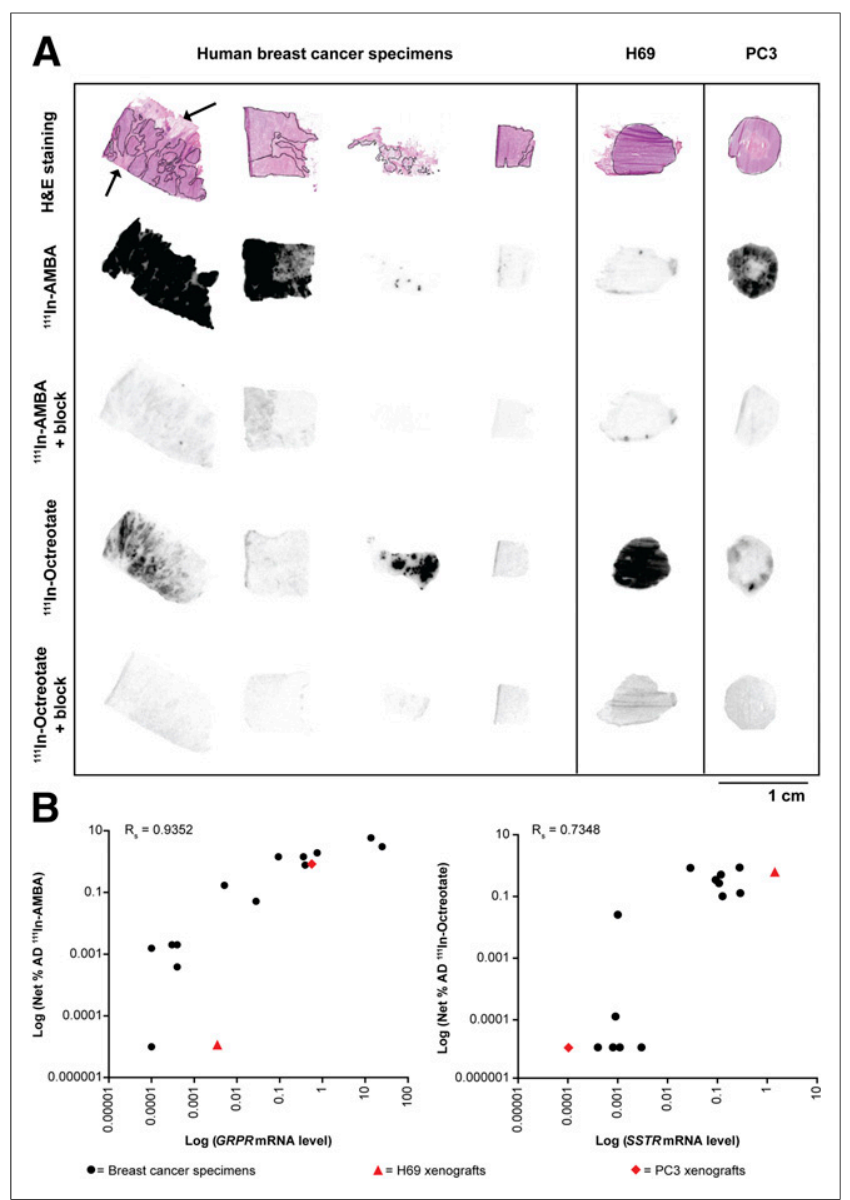

FIGURE 2. (A) In vitro autoradiography of human breast cancer specimens using ${ }^{111}$ In-AMBA (GRP analog) and ${ }^{111}$ In-DOTA-Tyr ${ }^{3}$-octreotate (SST analog) with and without block, demonstrating specific binding of radiotracers to receptor-positive tumor tissue. H69 (SSTR-positive, GRPR-negative) and PC3 xenografts (SSTR-negative, GRPR-positive) were used as controls. Tumor-containing areas are encircled in hematoxylin and eosin (H\&E) stainings. As example, arrows indicate nontumor-containing tissue in first H\&E staining. (B) Significant correlation between GRPR and SSTR2 mRNA levels and quantification of in vitro autoradiography results analyzed in 13 breast cancer specimens with variable receptor expression, demonstrating that mRNA levels of receptors can be used as predictor for radiotracer binding. $A D=$ added dose.

receptors can be used as a predictor for specific radiotracer binding. The CXCR4 radioligand pentaxifor, available to us, showed reduced receptor affinity when radiolabeled with ${ }^{111} \mathrm{In}$ for in vitro autoradiography purposes, hampering reliable in vitro autoradiography studies for CXCR4. Thus, studies correlating CXCR4 radiotracer binding and CXCR4 mRNA expression could not be performed. However, because Philip-Abbrederis et al. (19) reported on detecting CXCR4 mRNA expression in cell lines and successful in vivo imaging of corresponding xenograft models using ${ }^{68} \mathrm{Ga}$-pentaxifor, we concluded that $C X C R 4$ mRNA expression can also be used as a predictor for CXCR4 radioligand binding.

Concerning prognosis, we found no association between GRPR and SSTR2 expression and DFS, MFS, and OS in the M0 LNN patients. Surprisingly, we found that high CXCR4 levels correlated with better prognosis despite its negative correlation with ER, PR, and unfavorable GGI, indicating that a component of CXCR4 expression that is independent of these factors determines good outcome.
Other studies on CXCR4 expression in breast cancer have associated CXCR4 expression with poor patient survival (16). The discrepancy in study outcome might be explained by the fact that in our study we analyzed mRNA expression of the receptors (independent of receptor localization), whereas in the study by Salvucci et al. (16) tissue microarrays were analyzed by immunohistochemistry and nuclear and cytoplasmatic CXCR4 staining were analyzed separately. In agreement with our study, Salvucci et al. (16) reported more cytoplasmic CXCR4 staining in ER-negative (54\%) than ERpositive tumors (38\%).

Furthermore, we found that high GRPR expression was of modest predictive value for increased time to progression on tamoxifen treatment, suggesting GRPR radioligands to be useful in monitoring tumor response to treatment with tamoxifen. Recently, preclinical ${ }^{68} \mathrm{Ga}$-AMBA PET imaging in a mouse model also demonstrated the feasibility for monitoring tumor response after treatment with tamoxifen (27).

For the association with clinicopathologic and biologic characteristics analyzed in the M0 LNN patients, we observed a significant positive correlation between GRPR and SSTR2 expression and ESRI- and PGR-positive tumors. In line with our findings, significant positive correlation between SSTR2 and ER expression was reported previously (28), whereas van den Bossche et al. (29) reported estrogen-mediated regulation of SSTR2 expression in breast cancer cell lines. Because ESRI and PGR positivity correlates with breast cancer of the luminal subtype (2), tumors of this subtype could benefit most from GRPR- or SSTR2-mediated imaging or therapy. Moreover, ESRl-negative tumors showed low to no GRPR expression, and thus patients with ESRl-negative primary tumors are likely not suited for the application of GRPR radioligands. Because ESR1- or PGR-positive tumors account for $75 \%$ of the breast cancer tumors (2), GRPR- and SSTR2-mediated imaging and therapy might be of benefit for the larger part of the breast cancer patient population.

Concerning therapy, GRPR or SSTR2 radioligands can especially be of benefit for patients with ESRl-positive tumors who have progressed on various lines of endocrine treatment, because nearly all patients with recurrent disease become resistant against current antiestrogen treatments (4).

Previous studies we performed on GRPR and SSTR2 expression in human breast cancer specimens showed GRPR expression in 48

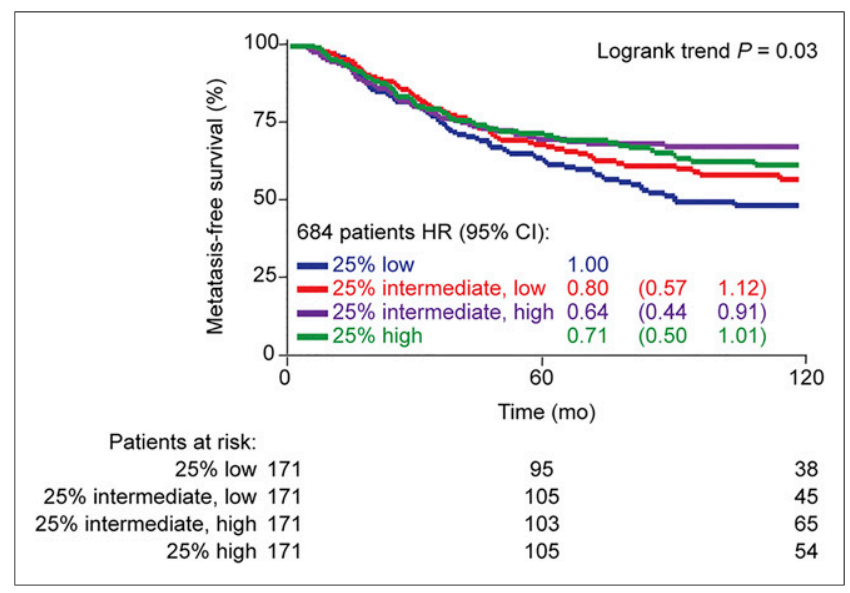

FIGURE 3. Distant MFS in 684 LNN patients as function of levels of CXCR4. $\mathrm{Cl}=$ confidence interval; $\mathrm{HR}=$ hazard ratio. 


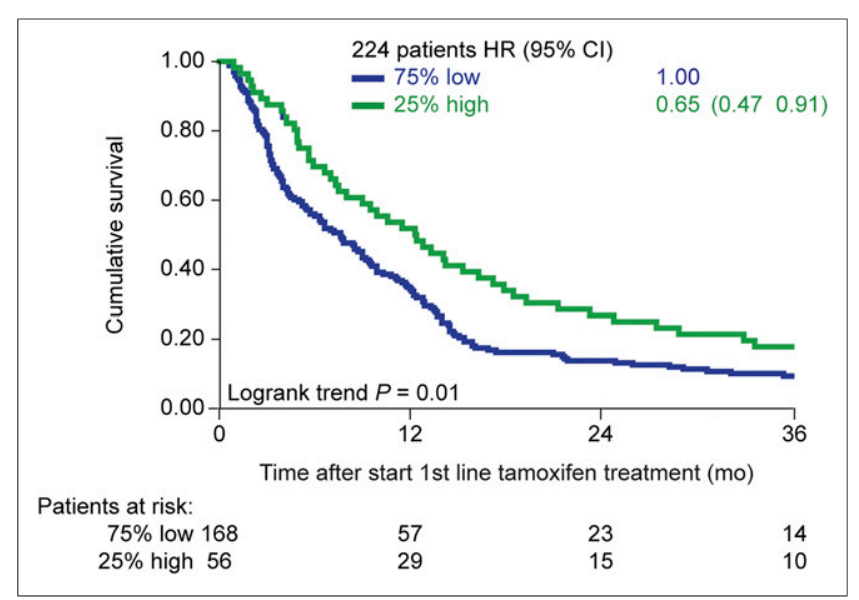

FIGURE 4. Association of GRPR expression with PFS on first-line tamoxifen treatment. $\mathrm{Cl}=$ confidence interval; $\mathrm{HR}=$ hazard ratio.

of 50 (30) and SSTR2 expression in 26 of 53 (SU Dalm, CHM van Deurzen, M Melis, M de Jong, unpublished data, 2014) of the specimens analyzed by in vitro autoradiography, emphasizing that GRPR- and SSTR2-mediated imaging and therapy could be applied in a large group of breast cancer patients.

Contrary to GRPR and SSTR2, high CXCR4 mRNA expression was correlated with ESRI- and PGR-negative tumors, associated with breast cancer of the basal like subtype (2), indicating that these tumors, in particular, might be suitable for CXCR4-mediated imaging or therapy. Patients with triple-negative tumors, especially, might benefit from CXCR4-mediated therapy, because effective therapy options for this aggressive subtype of breast cancer are scarce. Differences in CXCR4 expression between ESRI- and $P G R$-negative and ESRI- and $P G R$-positive patients were less pronounced than for GRPR and SSTR2. ESR1- and PGR-positive patients should therefore not be ruled out for CXCR4-mediated imaging or therapy.

Except for the presence of the receptors, for the selection of patients for imaging or treatment with radioligands, also the density of GRPR, SSTR2, and CXCR4 might determine the target of choice. In a study by Reubi et al. (15), among other receptors, GRPR and SSTR2 expression in 77 breast cancer tissues was analyzed using in vitro autoradiography. Results showed that high-density GRPR expression was observed in 50 of 77 tumors, compared with 14 of 77 tumors with high-density SSTR2 expression. Similarly, in our previous work we found homogeneous GRPR expression in $56 \%$ of the breast cancer specimens analyzed (30), whereas homogeneous SSTR2 expression was seen in $29 \%$ only (SU Dalm, CHM van Deurzen, M Melis, M de Jong, unpublished data, 2014).

One of the benefits of targeted imaging and therapy using GRPR, SSTR2, and CXCR4 radioligands is the possibility to upfront select patients who could benefit from these methods using one of the radioligands. For this, either frozen material from breast cancer biopsies can be used to perform in vitro autoradiography with radioligands or formalin-fixed paraffin-embedded material can be used for immunohistochemistry, or both can be used to perform RT-qPCR, to identify patients suited for imaging or therapy.

There are, however, also limitations to our study. First, mRNA expression was used as a surrogate for radiotracer binding and ER, PGR, and HER2 protein expression, which may, despite our current and previously published data $(22,23)$, turn out not to be entirely equivalent with protein expression. Second, for the prognostic part only, even though our study was relatively large no independent validation was performed. In addition, this is a retrospective study and might not completely represent the current situation in patients.

\section{CONCLUSION}

We successfully identified potential breast cancer patient groups for the application of radioligands targeting GRPR, SSTR2, or CXCR4 by analyzing associations between receptor expression and clinicopathologic, biologic, and prognostic factors. Our data show compelling evidence that sensitive and specific nuclear medicine-based imaging and therapy using radioligands might be of great benefit for selected breast cancer patients in a personalized setting. GRPR and SSTR2 radioligands in ER-positive and PRpositive tumors and CXCR4 radioligands in ER-negative patients might offer new, promising tools for imaging and therapy of breast cancer.

\section{DISCLOSURE}

The costs of publication of this article were defrayed in part by the payment of page charges. Therefore, and solely to indicate this fact, this article is hereby marked "advertisement" in accordance with 18 USC section 1734 . This study was funded by the Erasmus MC grant "The application of radiolabeled peptides in Breast Cancer" and the Cancer Genomics Netherlands funded by the Netherlands Organization for Scientific Research. No other potential conflict of interest relevant to this article was reported.

\section{REFERENCES}

1. International Agency on Research for Cancer. GLOBOCAN 2012: estimated cancer incidence, mortality and prevalence worldwide in 2012. International Agency on Research for Cancer website. http://globocan.iarc.fr/Default.aspx. Accessed August 18, 2015.

2. Yersal O, Barutca S. Biological subtypes of breast cancer: prognostic and therapeutic implications. World J Clin Oncol. 2014;5:412-424.

3. Brufsky AM. Current approaches and emerging directions in HER2-resistant breast cancer. Breast Cancer (Auckl). 2014;8:109-118.

4. Milani A, Geuna E, Mittica G, Valabrega G. Overcoming endocrine resistance in metastatic breast cancer: current evidence and future directions. World J Clin Oncol. 2014;5:990-1001.

5. Garcia EM, Storm ES, Atkinson L, Kenny E, Mitchell LS. Current breast imaging modalities, advances, and impact on breast care. Obstet Gynecol Clin North Am. 2013;40:429-457.

6. Mahoney MC, Newell MS. Screening MR imaging versus screening ultrasound: pros and cons. Magn Reson Imaging Clin N Am. 2013;21:495-508.

7. Hubbard RA, Kerlikowske K, Flowers CI, Yankaskas BC, Zhu W, Miglioretti DL. Cumulative probability of false-positive recall or biopsy recommendation after 10 years of screening mammography: a cohort study. Ann Intern Med. 2011;155:481-492.

8. Bison SM, Konijnenberg MW, Melis M, et al. Peptide receptor radionuclide therapy using radiolabeled somatostatin analogs: focus on future developments. Clin Transl Imaging. 2014;2:55-66.

9. Laznicek M, Laznickova A, Maecke HR. Receptor affinity and preclinical biodistribution of radiolabeled somatostatin analogs. Anticancer Res. 2012;32: 761-766.

10. Skånberg J, Ahlman H, Benjegard SA, et al. Indium-111-octreotide scintigraphy, intraoperative gamma-detector localisation and somatostatin receptor expression in primary human breast cancer. Breast Cancer Res Treat. 2002;74: $101-111$.

11. Van Den Bossche B, Van Belle S, De Winter F, Signore A, Van de Wiele C. Early prediction of endocrine therapy effect in advanced breast cancer patients using ${ }^{99 m}$ Tc-depreotide scintigraphy. J Nucl Med. 2006;47:6-13.

12. Reubi JC, Maecke HR. Peptide-based probes for cancer imaging. J Nucl Med. 2008;49:1735-1738. 
13. Wieser G, Mansi R, Grosu AL, et al. Positron emission tomography (PET) imaging of prostate cancer with a gastrin releasing peptide receptor antagonistfrom mice to men. Theranostics. 2014;4:412-419.

14. Bodei L, Ferrari M, Nunn AD, et al. ${ }^{177}$ Lu-AMBA bombesin analogue in hormone refractory prostate cancer patients: a phase I escalation study with singlecycle administrations [abstract]. Eur J Nucl Med Mol Imaging. 2007;34:S221.

15. Reubi C, Gugger M, Waser B. Co-expressed peptide receptors in breast cancer as a molecular basis for in vivo multireceptor tumour targeting. Eur J Nucl Med Mol Imaging. 2002;29:855-862.

16. Salvucci O, Bouchard A, Baccarelli A, et al. The role of CXCR4 receptor expression in breast cancer: a large tissue microarray study. Breast Cancer Res Treat. 2006;97:275-283.

17. Hanaoka H, Mukai T, Tamamura H, et al. Development of a ${ }^{111} \mathrm{In}$-labeled peptide derivative targeting a chemokine receptor, CXCR4, for imaging tumors. Nucl Med Biol. 2006;33:489-494.

18. Demmer O, Dijkgraaf I, Schumacher U, et al. Design, synthesis, and functionalization of dimeric peptides targeting chemokine receptor CXCR4. J Med Chem. 2011;54:7648-7662.

19. Philipp-Abbrederis K, Herrmann K, Knop S, et al. In vivo molecular imaging of chemokine receptor CXCR4 expression in patients with advanced multiple myeloma. EMBO Mol Med. 2015;7:477-487.

20. Jansen MP, Sieuwerts AM, Look MP, et al. HOXB13-to-IL17BR expression ratio is related with tumor aggressiveness and response to tamoxifen of recurrent breast cancer: a retrospective study. J Clin Oncol. 2007;25:662-668.

21. Sieuwerts AM, Lyng MB, Meijer-van Gelder ME, et al. Evaluation of the ability of adjuvant tamoxifen-benefit gene signatures to predict outcome of hormonenaive estrogen receptor-positive breast cancer patients treated with tamoxifen in the advanced setting. Mol Oncol. 2014;8:1679-1689.

22. Toussaint J, Sieuwerts AM, Haibe-Kains B, et al. Improvement of the clinical applicability of the Genomic Grade Index through a qRT-PCR test performed on frozen and formalin-fixed paraffin-embedded tissues. BMC Genomics. 2009; $10: 424$.

23. van Agthoven T, Sieuwerts AM, Meijer-van Gelder ME, et al. Relevance of breast cancer antiestrogen resistance genes in human breast cancer progression and tamoxifen resistance. J Clin Oncol. 2009;27:542-549.

24. Sieuwerts AM, Usher PA, Meijer-van Gelder ME, et al. Concentrations of TIMP1 mRNA splice variants and TIMP-1 protein are differentially associated with prognosis in primary breast cancer. Clin Chem. 2007;53:12801288 .

25. De Blois E, Schroeder RJ, De Ridder CA, Van Weerden WM, Breeman WP, De Jong M. Improving radiopeptide pharmacokinetics by adjusting experimental conditions for bombesin receptor-mediated imaging of prostate cancer. $Q \mathrm{~J} \mathrm{Nucl}$ Med Mol Imaging. June 19, 2013 [Epub ahead of print].

26. de Blois E, Chan HS, Konijnenberg M, de Zanger R, Breeman WA. Effectiveness of quenchers to reduce radiolysis of ${ }^{111} \mathrm{In}$ - or ${ }^{177} \mathrm{Lu}$-labelled methionine-containing regulatory peptides: maintaining radiochemical purity as measured by HPLC. Curr Top Med Chem. 2012;12:2677-2685.

27. Prignon A, Nataf V, Provost C, et al. ${ }^{68} \mathrm{Ga}-\mathrm{AMBA}$ and ${ }^{18} \mathrm{~F}-\mathrm{FDG}$ for preclinical PET imaging of breast cancer: effect of tamoxifen treatment on tracer uptake by tumor. Nucl Med Biol. 2015;42:92-98.

28. Kumar U, Grigorakis SI, Watt HL, et al. Somatostatin receptors in primary human breast cancer: quantitative analysis of mRNA for subtypes 1-5 and correlation with receptor protein expression and tumor pathology. Breast Cancer Res Treat. 2005;92:175-186.

29. Van Den Bossche B, D'Haeninck E, De Vos F, et al. Oestrogen-mediated regulation of somatostatin receptor expression in human breast cancer cell lines assessed with ${ }^{99 \mathrm{~m}}$ Tc-depreotide. Eur J Nucl Med Mol Imaging. 2004; 31:1022-1030.

30. Dalm SU, Martens JW, Sieuwerts AM, et al. In-vitro and in-vivo application of radiolabeled gastrin releasing peptide receptor ligands in breast cancer. $\mathrm{J} \mathrm{Nucl}$ Med. 2015;56:752-757. 